\title{
Laparoscopic Versus Conventional
} Open Rectum Amputation: a Clinical, Intraoperative, and Short-term Outcome Comparative Study

\author{
Előd Etele Élthes, Alexandra Lavinia Cozlea², Árpád Török \\ 1 2nd Department of Surgery, Mureș County University Hospital, Tîrgu Mureș, Romania \\ 2 Department of Gynecology, Mureș County University Hospital, Tîrgu Mureș, Romania
}

\section{CORRESPONDENCE}

\section{Előd Etele Élthes}

Str. Păcii nr. 68B/14

Tîrgu Mureș, Romania

Tel. +40746556355

E-mail: elthesetele@yahoo.com

\section{ARTICLE HISTORY}

Received: May 20, 2018

Accepted: June 12, 2018

\begin{abstract}
Objective: To evaluate and compare laparoscopic and conventional open rectum amputation procedures using clinical, intraoperative, postoperative, and oncological criteria. Methods: Fifty-nine patients with lower rectal and anorectal cancer were included in a retrospective study, conducted between 2014 and 2017. Patients underwent open or laparoscopic rectum amputation surgery and were divided into two groups: group 1 - laparoscopic amputation group (LAG) and group 2 - open amputation group (OAG). The clinical, intraoperative, and postoperative outcomes and oncological results were compared between the two groups. Results: We found a significantly smaller intraoperative blood loss $(325 \mathrm{~mL}$ vs. $538.29 \mathrm{~mL}$, $p=0.0002$ ), earlier return of bowel motility ( 2.41 days vs. 3.10 days, $p=0.036$ ), shorter hospital stays (10.08 days vs. 12.66 days, $p=0.03$ ), and a higher number of lymph nodes removed during surgery (12.33 nodes for LAG vs. 9.98 nodes for OAG, $p=0.049$ ). In the open surgery group we found shorter durations of surgery (199.58 minutes for LAG vs. 157.87 minutes for OAG, $p=0.0046)$. Conclusion: Laparoscopic rectum amputation is a technically demanding procedure. The present study demonstrates the benefits and disadvantages of this surgery, with comparable clinical, intraoperative, postoperative, and oncological results compared to the conventional open rectum amputation procedure.
\end{abstract}

Keywords: rectal tumor, laparoscopic, open, rectum amputation, oncologic

\section{INTRODUCTION}

Minimally invasive surgical techniques have revolutionized colorectal surgery in the last years, though it is still a rarely performed intervention. In addition, laparoscopic surgery for lower rectal and anorectal cancer is much more challenging compared to colon cancer, the main reason being the narrow space within the pelvis. Concerns about oncological radicality and long-term outcomes have limited the use of this type of operation. However, recent results of randomized 
controlled trials conducted on large numbers of patients have proven that laparoscopic surgery in the treatment of colorectal malignant diseases is safe from an oncological standpoint, and it also presents favorable short-term outcomes. ${ }^{1-4}$

\section{MATERIALS AND METHODS}

We conducted a retrospective study between 2014 and 2017, which included 59 patients with lower rectum and anorectal malignant tumors. For all patients included in this study, rectum amputation surgery was performed. Patients were selected and divided into two groups based on the surgical approach, as follows: group 1 - laparoscopic rectum amputation group (LAG), which included 12 patients, and group 2 - open rectum amputation group (OAG), with 47 patients. In order to compare the two types of procedures, clinical, intraoperative, postoperative, and oncological characteristics were evaluated.

The analyzed clinical characteristics included patient gender, age, tumor location, preoperative irradiation and/ or chemotherapy, severity of anemia at admission. The analyzed intraoperative characteristics comprised the duration of surgery, conversion rate, inoperability, presence of hepatic and/or pulmonary metastasis, local tumor invasion (prostatic, bladder, vaginal, sacral, perineal), intraoperative blood loss. Postoperative data compared between the two groups included the return of bowel motility, use of painkillers, length of hospital admission, death during hospital stay, and complications. Oncological outcomes were analyzed by comparing histopathological results, stadialization, removed lymph nodes, recurrence, and survival rates at 6,12 , and 18 months. Furthermore, the study investigates possible associations between anemia at admission and tumor stage in patients with and without preoperative oncological treatment, local invasion se- verity score, and operation time/hospital stay/complications. The local invasion of the tumor was estimated with the local invasion severity score, using points from 1 to 3 as follows: patients with one local invasion area -1 point; patients with two invasion areas -2 points; and patients with at least three local invasion areas -3 points. To assess the preoperative oncological treatment, the following scoring scale was applied: patients who underwent preoperative chemotherapy - 1 point; those who underwent preoperative irradiation -2 points; and patients who underwent both chemo- and radiotherapy - 3 points. For tumor staging (Dukes staging system), we used the following numbering scale: $\mathrm{A}=0, \mathrm{~B} 1=1, \mathrm{~B} 2=2, \mathrm{~B} 3=3, \mathrm{C} 1=4$, $\mathrm{C} 2=5, \mathrm{C} 3=6$. Patient data was statistically processed and analyzed using the GraphPad InStat software (GraphPad Software, California, USA), and the statistical significance was set at a value of $\mathrm{p}<0.05$.

\section{RESULTS}

Between 2014 and 2017, 59 patients diagnosed with lower rectal and anorectal cancer underwent open or laparoscopic rectum amputation surgery.

Table 1 presents the clinical characteristics of the patients. Most patients included in this study were diagnosed with lower rectal cancer (66\%). Fifteen patients underwent preoperative radiotherapy and 5 patients underwent preoperative chemotherapy. Only 5 patients benefited from both preoperative chemo- and radiotherapy.

Aspects regarding intraoperative and postoperative outcomes are presented in Table 2. In total, 47 patients underwent open rectum amputation and 12 patients underwent laparoscopic surgery. The duration of surgery was significantly longer for patients undergoing the laparoscopic procedure $(\mathrm{p}=0.0046)$. Conversion from laparoscopic to open surgery was considered necessary for one patient

TABLE 1. Clinical assessment

\begin{tabular}{lcc}
\hline & $\begin{array}{c}\text { Group 1 } \\
\text { Laparoscopic rectum } \\
\text { amputation } \\
\mathbf{n = 1 2}\end{array}$ & $\begin{array}{c}\text { Group 2 } \\
\text { Open rectum amputation } \\
\mathbf{n}=\mathbf{4 7}\end{array}$ \\
\hline Median age (years) & 65.29 & 63.92 \\
Gender ratio (F:M) & $9: 3$ & $18: 29$ \\
Tumor location (1/3 inferior: anorectal) & $9: 3$ & $36: 11$ \\
Median hemoglobin levels (g/dL) & 12.02 & 12.48 \\
Median hematocrit (\%) & 35.6 & 37.09 \\
Preoperative chemotherapy (yes: no) & $0: 12$ & $5: 42$ \\
Preoperative radiotherapy (yes: no) & $1: 11$ & $14: 33$ \\
\hline
\end{tabular}


TABLE 2. Intraoperative and postoperative outcomes

\begin{tabular}{lccc}
\hline & $\begin{array}{c}\text { Group } \mathbf{1} \\
\text { Laparoscopic rectum } \\
\text { amputation } \\
\mathbf{n = 1 2}\end{array}$ & $\begin{array}{c}\text { Group 2 } \\
\text { Open rectum amputation } \\
\mathbf{n = 4 7}\end{array}$ & p value \\
\hline Mean duration of surgery (minutes) & 199.58 & 157.87 & 0.0046 \\
Conversion (to open surgery) & 1 & 0 & - \\
Inoperability & 0 & 2 & - \\
Mean volume of blood loss (mL) & 325 & 538.29 & - \\
Hepatic metastasis & 0 & 2 & - \\
Prostatic invasion & 2 & 8 & - \\
Bladder invasion & 0 & 5 & - \\
Vaginal invasion & 1 & 9 & - \\
Sacral invasion & 1 & 10 & - \\
Perineal invasion & 2 & 6 & 0.036 \\
Return of bowel motility (days) & 2.41 & 3.10 & 0.03 \\
Mean hospital stay (days) & 10.08 & 12.66 & NS \\
Use of painkillers (days) & 7.66 & 8.02 & NS \\
Complications & 3 & 9 & - \\
Death during hospital stay & 1 & 1 & - \\
\hline
\end{tabular}

NS: non-significant $\mathrm{p}$ value $(>0.05)$

$(8.33 \%)$; the reason of conversion was non-surgical; there were serious alterations of vital functions caused by the Trendelenburg position during laparoscopic surgery. Inoperability was found in 2 patients (3.38\%); both presented pulmonary and hepatic metastases and advanced local invasion of the tumor. Intraoperative blood loss was significantly lower for patients in the LAG $(p=0.0002)$. The OAG included a higher number of patients with hepatic metastasis and more advanced local invasion of the tumor. The return of bowel motility was shorter for patients who underwent laparoscopic rectum amputation surgery ( $\mathrm{p}=$ 0.036 ). The average hospital stay was 11 days for the overall study population. A significantly shorter hospital stay was registered in case of patients in the LAG $(p=0.03)$. Painkillers were used for an average period of 7.84 days. Postoperative complications occurred in 3 cases from the LAG (bowel obstruction, parastomal hernia) and in 9 cases from the OAG (bowel obstruction, bladder fistula, ventral hernia, pelvic hematoma, perineal abscess). Death during hospital stay occurred in two cases, one for each type of procedure.

Data regarding oncologic outcomes and survival are presented in Table 3. There was a significant difference regarding the number of removed lymph nodes between the two surgical procedures. Patients in the LAG had more lymph nodes removed ( $p=0.049)$ compared to those in the OAG. Recurrence was present in one case in the LAG and 6 cases in the OAG. The average survival rate was 12 months. Furthermore, the statistical analysis of the tumor staging in patients with and without preoperative oncologic treatment resulted in an extremely significant moderate negative association for the OAG $(\mathrm{p}=0.0001)$.

TABLE 3. Oncological outcomes and prognosis

\begin{tabular}{lccc}
\hline & $\begin{array}{c}\text { Laparoscopic rectum am- } \\
\text { putation group (LAG) }\end{array}$ & $\begin{array}{c}\text { Open rectum amputation } \\
\text { group (OAG) }\end{array}$ & p value \\
\hline Histopatology (malignant:benign) & $12: 0$ & $46: 1$ & - \\
Dukes staging & 2.08 & 2.80 & $\mathrm{NS}$ \\
Outtaken lymphondes (n) & 12.33 & 9.98 & 0.049 \\
Recurrence & $1(8.33 \%)$ & $6(12.76 \%)$ & $\mathrm{NS}$ \\
Average survival rate (12 months) & $11(91.66 \%)$ & $44(93.61 \%)$ & $\mathrm{NS}$ \\
\hline
\end{tabular}

NS: not significant $\mathrm{p}$ value $(>0.05)$ 


\section{DISCUSSIONS}

In this study, patients were diagnosed with tumors situated in the lower rectum or anorectal region, the surgical procedure in this kind of tumors being extremely demanding. ${ }^{5}$ Being on the ascending part of the learning curve of laparoscopic rectum amputation surgery, we focused on case selection. Thus, the LAG had only 12 patients, while the OAG had 47 patients. Furthermore, the OAG presented more patients with hepatic metastasis, more advanced local invasion, more advanced tumor stages (Dukes B, C), and severe comorbidities in comparison with patients from the LAG. Preoperative oncologic treatment was performed in 25 patients (42.37\%). Preoperative radiotherapy reduces the rate of local recurrence, downstages and downsizes the tumor, consequently increasing the possibility of radical surgery. 6,7 The duration of surgery was longer for patients who underwent laparoscopic rectum amputation surgery. Other authors reported similar results. ${ }^{8-10}$ Intraoperative blood loss was significantly lower in case of patients who had undergone LAG compared to patients who underwent open surgical procedure, similar results being reported by other authors. ${ }^{8,10,12}$ Patients with laparoscopic rectum amputation surgery presented a significantly higher number of removed lymph nodes in comparison to patients from the OAG, and a recent study registered similar results. ${ }^{10,13}$ The return of bowel motility was significantly faster in case of patients from the LAG. Similar results were found in the current literature. ${ }^{8,12}$ The average hospital stay was 11 days for the overall study population. A significantly shorter hospital stay was registered in case of patients who underwent laparoscopic surgery, these data being in accordance with other reported results. ${ }^{9,10,16}$ Conversion to open surgery was required in only one patient (8.33\%). Recent studies registered similar results regarding conversion from a laparoscopic to an open approach. ${ }^{11,13,15}$ The overall postoperative complication rate was $20.33 \%$ in this study, other authors reporting similar results. . $^{3,14}$

\section{CONCLUSIONS}

Although laparoscopic rectum amputation is a technically demanding procedure, according to our study, a laparoscopic procedure for the treatment of lower rectum and anorectal malignant tumors is a feasible choice, resulting in less intraoperative blood loss, more excised lymph nodes, earlier return of bowel motility, and shorter periods of hospital admission. In addition, the laparoscopic procedure has shown similar results with the conventional open procedure regarding the postoperative use of painkillers, number of complications, recurrence, and survival. Our study confirms that the laparoscopic procedure can be a safe choice for the treatment of lower rectum and anorectal malignant tumors, offering better or at least equivalent results in comparison to the conventional open procedure.

\section{CONFLICT OF INTEREST}

None to declare.

\section{REFERENCES}

1. Fleshman J, Sargent DJ, Green E, et al. Laparoscopic colectomy for cancer is not inferior to open surgery based on a 5-year data from the COST study group trial. Ann Surg. 2007;246:655-662.

2. Hazebroek EJ, Color Study Group. COLOR: a randomized clinical trial comparing laparoscopic and open resection for colon cancer. Surg Endosc. 2002;16:949-953.

3. Guillo PJ, Quirke P, Thorpe H, et al. Short-term endpoints of conventional versus laparoscopic-assisted surgery in patients with colorectal cancer (MRC CLASICC trial); multicenter, randomized, controlled trial. Lancet 2005;365:1718-1726.

4. van der Pas MH, Haglind E, Cuesta MA, et al. Laparoscopic versus open surgery for rectal cancer (COLOR II): short-term outcomes of a randomized, phase 3 trial. Lancet Oncol. 2013;14:210-218.

5. Gouvas N, Tsiaoussis N, Pechlivanides G, et al. Laparoscopic or open surgery for the cancer of the middle and lower rectum: short-term outcomes of a comparative non-randomized study. Int J Colorectal Dis. 2009;24:761-769.

6. Sauer R, Becker H, Hohenberger W, et al. Preoperative versus postoperative chemoradiotherapy for rectal cancer. $N$ Engl J Med. 2004;351:1731-1740

7. Bujko K, Nowacki MP, Nasierowska-Guttmejer A, et al. Sphincter preservation following preoperative radiotherapy for rectal cancer: report of a randomized trial comparing short-term vs fractionated radiochemotherapy. Radiother Oncol. 2004;72:15-24.

8. Wen-Xi Wu, Yao-Min Sun, Yi-Bin Hua, et al. Laparoscopic versus conventional open resection of rectal carcinoma: A clinical comparative study. World J Gastroenterol. 2004; 10:1167-1170.

9. Sun Z, Kim J, Adam MA, et al. Minimally invasive versus open low anterior resection: Equivalent survival in a national analysis of 14,033 patients with rectal cancer. Ann Surg. 2016;263:1152-1158.

10. Boutros M, Hippalgaonkar N, Silva E, et al. Laparoscopic resection of rectal cancer results in higher lymph node yield and better short-term outcomes than open surgery: a large single-center comparative study. Dis Colon Rectum. 2003; 56:679-688.

11. Krane MK, Fichera A. Laparoscopic rectal cancer surgery: where do we stand? World J Gastroenterol. 2012;18:6747-6755.

12. Chand M, Bhoday J, Brown G, et al. Laparoscopic surgery for rectal cancer. J R Soc Med. 2012;105:429-435.

13. Nussbaum DP, Speicher PJ, Ganapathi AM, et al. Laparoscopic versus open low anterior resection for rectal cancer: results from the nationa cancer data base. J Gastrointest Surg. 2015;19:124-131.

14. Indar A, Efron J. Laparoscopic surgery for rectal cancer. Perm J. 2009;13:47-52.

15. Reza MM, Blasco JA, Andradas E, et al. Systematic review of laparoscopic versus open surgery for colorectal cancer. Br J Surg. 2006;93:921-928.

16. Miyajima N, Fukunaga $M$, Hasegawa $H$, et al. Results of a multicenter study of 1057 cases of rectal cancer treated by laparoscopic surgery. Surg Endosc. 2009;23:113-118. 